\title{
Epidemiological Characterization of Dermatomycosis in Ethiopia
}

This article was published in the following Dove Press journal:

Clinical, Cosmetic and Investigational Dermatology

\author{
Shambel Araya (iD \\ Million Abuye \\ Abebe Edao Negesso \\ Addis Ababa University College of Health \\ Sciences, Department of Medical \\ Laboratory Sciences, Addis Ababa, \\ Ethiopia
}

Background: Superficial mycosis is common worldwide and their epidemiological characteristics are different in different geographical areas and have shown variations in the last decades. The aim of this study was to analyze and characterize the epidemiology of dermatomycosis and their causative fungi species in Ethiopia between 2015 and 2019.

Methods: A laboratory-based cross-sectional study was conducted using the data of mycological examination and culture findings from all patients who visited the Dermatology Department of Arsho Advanced Medical Laboratory, Addis Ababa, Ethiopia. The direct wet mount microscopy and culture data of the isolates were collected from the database of the dermatology unit from 2015 to 2019 after permission was obtained from the laboratory head. The data were double-entered into Microsoft Excel, exported and analyzed using SPSS version 20.

Results: The total prevalence of fungi causing dermatomycosis was $67.7 \%$ (760/1122 cases) using direct wet mount microscopy and from these 489/1122 (43.5\%) were culture positive. Dermatomycosis was found to be higher among females 694/1122 (61.9\%) than male participants. Age group 25-44 years was the most affected 442/1122 (39.4\%) followed by $1-14$ years old $291 / 1122(25.94 \%)$. Tinea unguium $(50.8 \%)$ is the most common type of dermatomycosis followed by tinea capitis $(24.1 \%)$ and tinea corporis (13.9\%). Trichophyton spp. (32\%) was the most highly distributed causative agent, followed by Epidermophyton spp. (20.2\%) and Aspergillus fumigatus (8.3\%).

Conclusion: The retrospective analysis of epidemiological data collected at Arsho Advanced Medical Laboratory since 2015 showed a gradual increase in the frequency of tinea unguium and tinea pedis. However, during the past years, there was a gradual decline in the frequency of tinea corporis. In parallel with this variable pattern, the rate of isolation of non-dermatophytes especially Aspergillus fumigates and Candida species has shown a gradual increment during the past five years.

Keywords: dermatomycosis, dermatophytes, tinea, fungi, Addis Ababa, Ethiopia

\section{Introduction}

Superficial mycosis (dermatomycosis) is a disease of the nail, skin and hair caused by both dermatophytes and some non-dermatophyte fungal agents. Over the past years, the prevalence of superficial fungi infections has shown a gradual increase and they have affected $20-25 \%$ of the total world's population. ${ }^{1-3}$ Dermatomycosis is common in most tropical countries due to elevated humidity, elevated temperature, and sweating. The most common examples of superficial mycoses include dermatomycosis, candidiasis, and pityriasis versicolor. ${ }^{4-7}$

Dermatophytosis is mainly confined to the keratinized layers because its fungal agents are not able to penetrate into the organ or deeper tissue of healthy
Correspondence: Shambel Araya

Tel +251939459529

Email shambelaraya8@gmail.com

Clinical, Cosmetic and Investigational Dermatology 2021:14 83-89 
individuals. However, these kind's infections are also dependent on the immune status of the host, fungal agent and site of infection. ${ }^{8-11}$ Superficial mycoses can easily spread through fomites or direct contact with the infected humans and animals. Although the infection is curable and non-invasive, its widespread nature and therapeutic costs are major worldwide public health problems. ${ }^{12,13}$ The clinical lesions of superficial mycosis are highly variable and closely resemble other skin diseases. Therefore, it is important to have a confirmed laboratory diagnosis of superficial mycosis due to fungal agents. ${ }^{14-16}$

Currently, according to recent update on taxonomy of dermatophytes there are 52 identified species of dermatophytes in seven genera's. ${ }^{17}$ From these, around 20 species belong to the three most prevalent and recognized worldwide genera's; Trichophyton (16 species), Epidermophyton (1 spp.) and Microsporum (3 spp.) and these genera's are currently known to infect humans. ${ }^{17-19}$

Dermatomycosis affect more than $40 \%$ of the world's population, and tinea unguium accounts for approximately $18 \%-40 \%$ of the onychomycosis representing a worldwide public health problem. ${ }^{20}$ Ethiopia is located in a tropical region and it has a wet humid climate which makes it fall among the regions affected with dermatomycosis. However, the frequency and distribution of pathogenic species and the clinical manifestation vary with time and geographical location. ${ }^{21,22}$ For all the above mentioned reasons and problems, the aim of this study was to determine changes in distribution of dermatophytes and nondermatophytes, to compare species distribution according to demographic characteristics and body site of the patients in Addis Ababa, Ethiopia.

\section{Methods}

Retrospective study was used to include all cases of dermatophytosis visiting the mycology laboratory of Arsho Advanced Medical Laboratory from January 2015 to December 2019. Age, gender, clinical manifestations and site of infection were recorded for each subject from the laboratory database. For direct wet mount microscopy and culture investigation samples were collected from scalp, skin, nail clippings, plucked hairs, and sole scrapes. Samples used for direct wet mount microscopy were placed on glass slides and treated with a $10 \%$ w/v KOH. In Arsho Advanced Medical Laboratory mycology laboratory, the clinical sample was inoculated onto Sabouraud's dextrose agar (SDA) (Oxoid, Basingstoke, England) containing chloramphenicol with cycloheximide, and without cycloheximide, prepared based on manufacturer instructions. For identification of dermatophytes incubation at $27^{\circ} \mathrm{C}$ was performed for at least three weeks, and identification of yeasts at 5-7 days. Inoculated samples in SDA were checked twice a week for any presence of fungal growth. Cultures without evident fungal growth were kept for six weeks before it was considered as negative for fungi. Identification of fungi was based on microscopic and macroscopic characteristics of the colonies on SDA. Dermatophytes suspected colonies were sub-cultured into potato dextrose agar (Oxoid, Basingstoke, UK) for the presence of spores.

When a non-dermatophyte was found, it was considered as relevant only in the presence of spores, hyphae, or yeast cells on microscopic examination. In case of mold isolate; colony taken from SDA and PDA was confirmed by using lactophenol cotton blue stain.

Yeasts were identified using chromogenic medium, CHROMagar Candida (bioMérieux, France) and using routine conventional diagnostic methods as per the manufacturer instruction. The database of Arsho advanced diagnostic laboratory contains information concerning clinical aspects of dermatomycosis, age, sex, date of examination, direct microscopy and culture results. But some culture results were not to species level.

\section{Statistical Analysis}

Data were collected, double-entered, cleaned and analyzed using SPSS version 20 software according to the study objectives. Frequency and percentage were used for analysis of the outcome.

\section{Results}

A total of 1122 specimens were diagnosed for dermatomycosis from 2015-2019 in Arsho advanced diagnostic laboratory. Among these study participants, 694 (61.9\%) were females. The total prevalence of positive $\mathrm{KOH}$ was $67.7 \%$ while positive cultures among the total specimens received were $43.5 \%$.

Tinea unguium $(50.8 \%)$ was the most dominant form of superficial mycosis followed by tinea capitis (24.1\%) and tinea corporis (13.9\%). Over all, clinical manifestations were prevalent in female (694 (61.9\%) study participants with compared to male (Table 1).

Tinea unguium, tinea corporis and tinea pedis affects mostly adults $25-44$ years old. (442/1122), while tinea capitis (160/271) were higher among children's 160/271 
Table I Distributions of Superficial Mycosis in Relation to Sex Addis Ababa, Ethiopia $(n=1122)$

\begin{tabular}{|l|l|l|l|}
\hline Clinical Manifestation & $\begin{array}{l}\text { Male } \\
\mathbf{n ( \% )}\end{array}$ & $\begin{array}{l}\text { Female } \\
\mathbf{n}(\%)\end{array}$ & $\begin{array}{l}\text { Total } \\
\mathbf{n}(\%)\end{array}$ \\
\hline Tinea capitis (n (\%) & $95(35)$ & $176(65)$ & $27 I(24.1)$ \\
Tinea unguium(n (\%) & $200(35)$ & $371(65)$ & $571(50.8)$ \\
Tinea corporis(n (\%) & $74(47.1)$ & $83(52.8)$ & $157(13.9)$ \\
Tinea pedis (n (\%) & $44(50)$ & $44(50)$ & $88(7.8)$ \\
Tinea cruris (n (\%) & $15(42.9)$ & $20(57.1)$ & $35(3.1)$ \\
Total & $428(38.1)$ & $694(6 I .9)$ & $1122(100)$ \\
\hline
\end{tabular}

(59.4\%) and tinea cruris were more prevalent in elder patients age $>65$ year old 9/35 (25\%) (Table 2).

Among the dermatophytosis annual distribution from 2015 to 2017, the prevalence of tinea unguium increased from $42.7 \%(88 / 206)$ to $63 \%(140 / 222)(131 / 571)$ while tinea capitis decreased from $28.1 \%(58 / 206)$ to $16.66 \%$ (37/222) respectively (Figure 1). The frequency of dermatophytes had increased from $21.4 \%$ in 2015 to $64.8 \%$ in 2017 and then declined in 2018 and 2019 respectively (Figure 1).

Tinea uniguium was a predominant isolate for the past five years with a mean prevalence of $50.8 \%$ followed by tinea capitis $(24.1 \%)$ and tinea corporis $(13.9 \%)$. Tinea pedis shows a gradual increase starting from 2016-2019 (4\%-9.4\%). The highest frequency of tinea unguium was in the year 2017 (63.3\%) followed by 2016 (50.5\%) (Figure 2).

Trichophyton spp. and Epidermophyton spp. were associated with $52.4 \%$ of the culture positive specimens. Trichophyton spp. was the most dominant dermatophyte (32.1\%) found during the study period and this was the most common fungi agent in tinea unguium, tinea pedis, tinea manuum and tinea cruris respectively. Epidermphyton spp. and Aspergillus fumigates was the second and third most frequently isolated species respectively $(20.2 \%, 8.3 \%)$. Epidermophyton spp. was the commonest fungal agent in tinea corporis $(29.5 \%)$ (Table 3).

\section{Discussion}

Prevalence of dermatomycosis was found to be variable in various age groups and genders. Dermatomycosis can affect both genders. However, in this study we found a higher prevalence of dermatomycosis among female than males. This result has been reported by other authors in previous studies; ${ }^{23,24}$ although it is contradicted in other studies. ${ }^{13,25}$ Possible explanation for this could be due to females usage of costumes, which cause excessive sweating, frequent housework, inability to maintain good nail care and, cosmetic reasons could be a factor for the high occurrence of dermatomycosis among females.

Age was found to be a basic factor influencing the distribution of dermatophytosis on the studied population. There was high occurrence of dermatomycosis among study subjects with the of age 25-44 years old might be due to the fact that most adults on this age are active in working different jobs which might expose them to this infection. Some factors like smoking, diabetes and HIV/ AIDS could also play an important role in contracting dermatomycosis and exposure to the dermatophyte and non-dermatophytes. ${ }^{13,21,26}$ In our findings, tinea unguium was the most common (32\%) type of dermatomycosis infection relative to the other tinea types which is similar with other studies. This is similar to previous studies. ${ }^{27,28}$ Several authors suggest that inability to maintain good nail care and cosmetic reasons, reduced growth rate of the ungual plate, frequent housework, poor peripheral circulation and an increase in trauma rates could play an important role in this manifestation. ${ }^{25,28}$ The high prevalence of dermatomycosis on toenails and the importance of this site concerning dermatomycosis are reported in several studies. $^{25,28}$ These reports stated that the infection at this

Table 2 Frequency of Clinical Manifestation in Different Age Groups Addis Ababa, Ethiopia ( $\mathrm{n}=$ I I22)

\begin{tabular}{|c|c|c|c|c|c|c|c|}
\hline \multirow[t]{2}{*}{ Site } & \multicolumn{7}{|c|}{ Age Group (Years) } \\
\hline & $1-14$ & $15-24$ & $25-44$ & 45-64 & $\geq 65$ & Total & (\%) \\
\hline T. capitis & 160 & 26 & 69 & 8 & 8 & 271 & 24.1 \\
\hline T. corporis & 33 & 23 & 61 & 30 & 10 & 157 & 13.9 \\
\hline T. unguium & 83 & 92 & 264 & 106 & 26 & 571 & 50.8 \\
\hline T. pedis & 8 & 10 & 42 & 18 & 10 & 88 & 7.8 \\
\hline T. cruris & 7 & 5 & 6 & 8 & 9 & 35 & 3.1 \\
\hline Total & 291 & 156 & 442 & 170 & 63 & 1122 & $100 \%$ \\
\hline
\end{tabular}




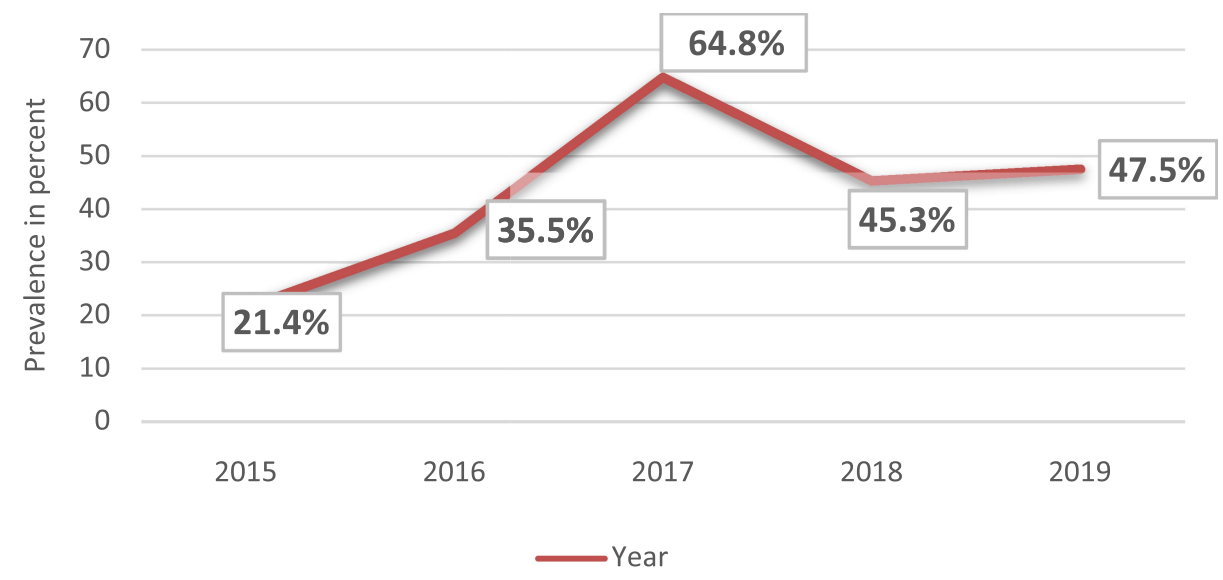

Figure I Trends of dermatophytes, Addis Ababa, Ethiopia.

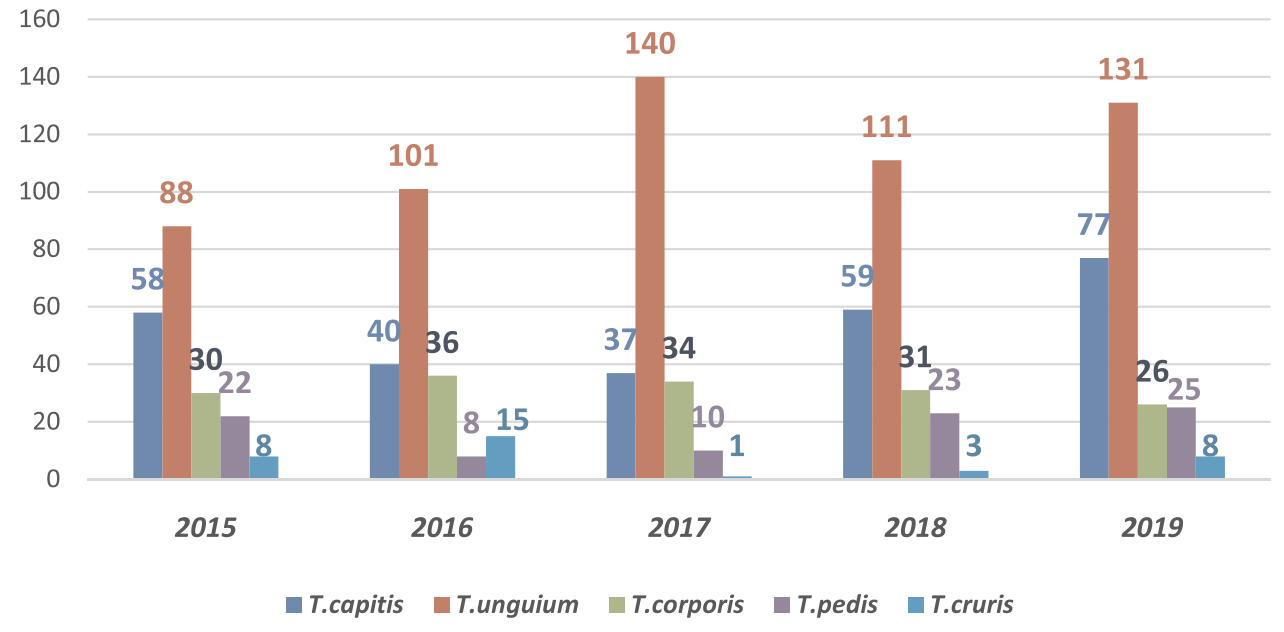

Figure 2 Epidemiology of dermatomycosis, Addis Ababa, Ethiopia.

site could be due to, lack of hygiene, use of shoes over long periods of time, lack of protection at work and poor circulation. Onychomycosis is usually considered of occupational nature, especially among activities that require frequent contact with water. In addition, the maceration of periungual tissue caused by the use of manicure tools can result cross-infection among people who use this service. $^{27,28}$

However, this finding is in contrast to other studies who reported tinea corporis, ${ }^{21}$ tinea capitis ${ }^{29}$ and tinea cruris $^{30}$ as the commonest clinical manifestations than tinea unguium. Tinea capitis is the second most common form of tinea and accounted $24.1 \%$ of all dermatomycosis. The frequency rate of tinea capitis was higher in females than males, the most common being the children under age 14 years old. And this might be due to the fact that children have the inadequacy of natural protective fatty acids synthesized in their scalp among prepubescent children. Different studies have also explained the above factor for the high occurrence of tinea capitis in children. ${ }^{21,31-33}$

In our study, Trichophyton spp. (31\%), Epidermophyton spp. (16.6\%) and Cladosporium spp. (13.3\%) were the major fungi causing tinea capitis that is similar with studies conducted in Ethiopia. ${ }^{21,22,34}$ Tinea cruris was the least form of superficial mycosis and accounted for $3.1 \%$ of all tineas. Many people with tinea cruris have coincident tinea pedis, and it has been reported that tinea cruris is transmitted by hand from the tinea pedis. ${ }^{30,31,35}$ The high incidence of tinea cruris was in the age group greater than 45 years old and females were mostly affected than males. In this retrospective study, Trichophyton spp. 40\% (6/15) followed by Cladosporium spp. 20\% (3/15) were the dominant fungi resulting in tinea cruris which is in line with studies conducted in Iran, India, and Nigeria. ${ }^{25,30,31}$ 
Table 3 Distribution of Dermatophytes and Non-Dermatophytes Fungi Isolates in Relation to Clinical Manifestation Addis Ababa, Ethiopia

\begin{tabular}{|c|c|c|c|c|c|c|c|}
\hline \multicolumn{2}{|l|}{ Isolates } & \multicolumn{5}{|c|}{ Clinical Manifestation } & \multirow[t]{2}{*}{ Total (n) } \\
\hline & & T. capitis & T. unguium & T. corporis & T. pedis & T. cruris & \\
\hline \multirow{3}{*}{$\begin{array}{l}\text { Dermatophytes } \\
n=28 I\end{array}$} & Trichophyton spp. & 28 & 95 & 15 & 13 & 6 & 157 \\
\hline & M. audouinii & 5 & 12 & 4 & 3 & 1 & 25 \\
\hline & Epidermophyton spp. & 15 & 59 & 18 & 5 & 2 & 99 \\
\hline \multirow[t]{12}{*}{ Molds $n=147$} & Cladosporium spp. & 12 & 14 & 5 & 3 & 3 & 33 \\
\hline & Fusarium spp. & 3 & 14 & 4 & 0 & 1 & 22 \\
\hline & Penicillium spp. & 2 & 14 & 0 & 0 & 0 & 16 \\
\hline & Scopulariopsis brevicaulis & 0 & 4 & 0 & 0 & 0 & 4 \\
\hline & Colletotrichum spp. & 1 & 0 & 1 & 1 & 0 & 3 \\
\hline & Acremonium spp. & 0 & 1 & 0 & 0 & 0 & I \\
\hline & Alternaria spp. & 0 & 3 & 0 & 0 & 0 & 3 \\
\hline & Paecilomyces spp. & 1 & 0 & 0 & 0 & 0 & I \\
\hline & Neoscytalidium dimidiatum & I & 11 & 1 & 0 & 0 & 13 \\
\hline & Aspergillus fumigates & 12 & 24 & 2 & 3 & 0 & 41 \\
\hline & Aspergillus niger & 0 & 3 & 1 & 1 & 0 & 5 \\
\hline & Aspergillus terreus & 0 & 1 & 0 & 0 & 0 & I \\
\hline \multirow[t]{5}{*}{ Yeasts $n=61$} & Candida albicans & 4 & 16 & I & I & I & 23 \\
\hline & Candida cruise & 4 & 10 & 6 & 0 & 0 & 20 \\
\hline & Candida glabrata & 2 & 7 & 0 & 0 & 0 & 9 \\
\hline & Rhodotorula mucilaginosa & 0 & 4 & 3 & 1 & 1 & 9 \\
\hline & Total & 90 & 292 & 61 & 31 & 15 & 489 \\
\hline
\end{tabular}

In this study, tinea corporis is the third common manifestation in prevalence and accounted for $13.9 \%$ of all tineas. Similar results were found in Ethiopia ${ }^{21,22}$ and in Egypt, ${ }^{36}$ India $^{30,37,}$ and Brazil. ${ }^{38,39}$ Whereas, Singh et al, ${ }^{30}$ Naseri et al, ${ }^{25}$ and Bhatia et $\mathrm{al},{ }^{40}$ found tinea corporis as the dominant form of dermatomycosis ranging from $33.1 \%-39.5 \%$. The most common being the age group 25-44 years old. In the current study Epidermophyton spp. (29.5\%), Trichophyton spp. (24.5\%) and Candidacruzie $(9.8 \%)$ were the main fungi causing tinea corporis respectively and this is in agreement with similar studies in Ethiopia, Nigeria and India. ${ }^{21,23,30,41,42}$

Non-dermatophytes molds were isolated from 143/489 (29.2\%) study subjects. With Aspergillus spp. as a major isolate accounting $41 / 143(28.67 \%)$ of the total non-dermatophyte molds isolates followed by Cladosporium spp. $33 / 143(23 \%)$ that is similar with studies done in Ethiopia. $^{21,22}$ Non-dermatophytes were isolated from culture positive clinical samples, nail and hair being the most affected body sites. The significance of such non-dermatophyte molds in causing onychomycosis has been reported in other studies. ${ }^{43}$ Similarly, yeasts were isolated $63 / 489(12.8 \%)$ from patients with $C$. albicans as a dominant isolate consisting 23/63 (37.7\%) of the total yeasts isolated and this current finding is similar to a studies conducted in Ethiopia. ${ }^{21,43,44}$ Candida albicans has been isolated mainly from nail infection. Candida albicans as major cause of tinea unguium has been reported in other publications. ${ }^{4,44}$

\section{Conclusion}

Dermatophytes can affect all age groups and genders. Dermatomycosis was higher among females than males except for fingernail infection. Adult' age group (25-44) was more affected by dermatomycosis than the other age groups. The high prevalence of tinea corporis occurred in males rather than in females. Further large studies on the prevalence of dermatomycosis, fungal etiological agents, and changes in species distribution of the etiological agents of superficial infection in Ethiopia are important. Currently there are limited studies on antifungal resistance status and molecular identification of fungi; hence molecular characterization and understanding their antifungal susceptibility pattern of fungi is recommended. 


\section{Data Sharing Statement}

All necessary data are included in the manuscript.

\section{Ethical Approval and Consent to Participate}

Ethical clearance was obtained from Medical Laboratory College of Health Sciences Addis Ababa University ethical review committee and was in accordance with the principles of the Helsinki II declaration. No informed consent was required as the research in this article related to retrospective analysis of micro-organisms and patient information was kept confidential. Data was collected after official permission was obtained from Arsho Advanced Diagnostic Laboratory head.

\section{Acknowledgments}

We are very grateful to Arsho Advanced Medical Laboratory for letting us access their database to collect the data. Addis Ababa University, College of Health Sciences is duly acknowledged for financial support.

\section{Funding}

There is no funding to report.

\section{Disclosure}

The authors declare that they have no financial or nonfinancial conflicts of interest for this work.

\section{References}

1. Brasch J, Glaser R. Dynamic diversity of dermatophytes. Hautarzt. 2019;70(8):575-580. doi:10.1007/s00105-019-4427-3

2. Ferguson L, Fuller LC. Spectrum and burden of dermatophytes in children. J Infect. 2017;74(Supp11):S54-S60. doi:10.1016/S0163-4453(17)30192-5

3. Bouchara JP, Mignon B, Chaturvedi V. Dermatophytes and dermatophytoses: a thematic overview of state of the art, and the directions for future research and developments. Mycopathologia. 2017;182(1-2):14. doi:10.1007/s11046-017-0114-z

4. Khurana A, Sardana K, Chowdhary A, et al. Clinical implications of antifungal drug susceptibility testing of dermatophytes. Indian Dermatol Online J. 2019;10(6):737-738. doi:10.4103/idoj.IDOJ_253_19

5. Pierard GE. Dermatomycoses due to dermatophytes. Revue Medicale De Liege. 2016;71(3):147-153.

6. White TC, Findley K, Dawson TL, et al. Fungi on the skin: dermatophytes and Malassezia. Cold Spring Harb Perspect Med. 2014;4(8): a019802-a019802. doi:10.1101/cshperspect.a019802

7. Cafarchia C, Iatta R, Latrofa MS, et al. Molecular epidemiology, phylogeny and evolution of dermatophytes. Infect Genet Evol. 2013;20:336-351. doi:10.1016/j.meegid.2013.09.005

8. Ali-Shtayeh MS, Khaleel T, Jamous RM. Ecology of dermatophytes and other keratinophilic fungi in swimming pools and polluted and unpolluted streams. Mycopathologia. 2002;156(3):193-205. doi:10.1023/A:1023311411004

9. Ng KP, Soo-Hoo TS, Na SL, et al. Dermatophytes isolated from patients in University Hospital, Kuala Lumpur, Malaysia. Mycopathologia. 2002;155(4):203-206. doi:10.1023/A:1021194025765
10. Ellabib MS, Khalifa ZM. Dermatophytes and other fungi associated with skin mycoses in Tripoli, Libya. Ann Saudi Med. 2001;21(34):193-195. doi:10.5144/0256-4947.2001.193

11. Seebacher C. Epidemiology, clinic and treatment of dermatomycoses caused by zoophilic dermatophytes. Mycoses. 2000;43(Suppl 1):4-7.

12. Cabanes FJ. Dermatophytes: the names they are a-changin. Rev Iberoam Micol. 2020;37(1):1-2. doi:10.1016/j.riam.2019.10.002

13. Kalita JM, Sharma A, Bhardwaj A, et al. Dermatophytoses and spectrum of dermatophytes in patients attending a teaching hospital in Western Rajasthan, India. J Family Med Prim Care. 2019;8 (4):1418-1421. doi:10.4103/jfmpc.jfmpc_159_19

14. Allahdadi M, Hajihossein R, Kord M, et al. Molecular characterization and antifungal susceptibility profile of dermatophytes isolated from scalp dermatophyte carriage in primary school children in Arak city, Center of Iran. J Mycol Med. 2019;29(1):19-23. doi:10.1016/j. mycmed.2019.01.002

15. Ali SY, Gajjala SR, Raj A. Study of prevalence of dermatophytes among human immunodeficiency virus/AIDS patients in Shadan Institute of Medical Sciences and Teaching Hospital and Research Centre, Hyderabad, Telangana, India. Indian J Sex Transm Dis AIDS. 2018;39(2):98-101. doi:10.4103/ijstd.IJSTD_103_16

16. da Cunha KC, Riat A, Normand A-C, et al. Fast identification of dermatophytes by MALDI-TOF/MS using direct transfer of fungal cells on ground steel target plates. Mycoses. 2018;61(9):691-697. doi:10.1111/myc. 12793

17. de Hoog GS, Dukik K, Monod M, et al. Toward a novel multilocus phylogenetic taxonomy for the dermatophytes. Mycopathologia. 2017;182(1-2):5-31. doi:10.1007/s11046-016-0073-9

18. Ameen M. Epidemiology of superficial fungal infections. Clin Dermatol. 2010;28(2):197-201. doi:10.1016/j.clindermatol.20 09.12.005

19. Ndako J, Osemwegie OO, Spencer TH, et al. Prevalence of dermatophytes and other associated fungi among school children. Global Advan Res J Med Med Sci. 2012;1(3):049-056.

20. Havlickova B, Czaika VA, Friedrich M. Epidemiological trends in skin mycoses worldwide. Mycoses. 2008;51(Suppl 4):2-15. doi:10.1111/j.1439-0507.2008.01606.x

21. Bitew A. Dermatophytosis: prevalence of dermatophytes and nondermatophyte fungi from patients attending Arsho advanced medical laboratory, Addis Ababa, Ethiopia. Dermatol Res Pract. 2018;2018:8164757. doi:10.1155/2018/8164757

22. Teklebirhan G, Bitew A. Prevalence of dermatophytic infection and the spectrum of dermatophytes in patients attending a Tertiary Hospital in Addis Ababa, Ethiopia. Int $J$ Microbiol. 2015;2015:653419. doi:10.1155/2015/653419

23. Colosi IA, Cognet O, Colosi HA, et al. Dermatophytes and dermatophytosis in Cluj-Napoca, Romania-A 4-year cross-sectional study. $J$ Fungi (Basel). 2020;6(3):154.

24. Das S, De A, Saha R, et al. The current Indian epidemic of dermatophytosis: a study on causative agents and sensitivity patterns. Indian J Dermatol. 2020;65(2):118-122. doi:10.4103/ijd.IJD_203_19

25. Ebrahimi M, Zarrinfar H, Naseri A, et al. Epidemiology of dermatophytosis in northeastern Iran; A subtropical region. Curr Med Mycol. 2019;5(2):16-21. doi:10.18502/cmm.5.2.1156

26. Hassanzadeh Rad B, Hashemi SJ, Farasatinasab M, et al. Epidemiological survey of human dermatophytosis due to zoophilic species in Tehran, Iran. Iran J Public Health. 2018;47(12):19301936.

27. Takenaka M, Murota H, Nishimoto K. Epidemiological survey of 42 403 dermatophytosis cases examined at Nagasaki University Hospital from 1966 to 2015. J Dermatol. 2020;47(6):615-621. doi:10.1111/ $1346-8138.15340$

28. Sadeghi Tafti H, Falahati M, Kordbacheh P, et al. A survey of the etiological agents of scalp and nail dermatophytosis in Yazd, Iran in 2014-2015. Curr Med Mycol. 2015;1(4):1-6. doi:10.18869/acadpub. cmm.1.4.1 
29. Jain S, Kabi S, Swain B. Current trends of dermatophytosis in Eastern Odisha. J Lab Physicians. 2020;12(1):10-14. doi:10.1055/s0040-1713063

30. Singh B, Tripathy T, Kar BR, et al. Clinicomycological study of dermatophytosis in a Tertiary Care Hospital in Eastern India: a cross-sectional study. Indian Dermatol Online J. 2020;11(1):46-50. doi:10.4103/idoj.IDOJ_62_19

31. Dalis JS, Kazeem HM, Kwaga JKP, et al. Prevalence and distribution of dermatophytosis lesions on cattle in Plateau State, Nigeria. Vet World. 2019;12(9):1484-1490. doi:10.14202/vetworld.2019.1484-1490

32. Pathania S, Rudramurthy S, Narang T, et al. A prospective study of the epidemiological and clinical patterns of recurrent dermatophytosis at a tertiary care hospital in India. Indian J Dermatol Venereol Leprol. 2018;84(6):678-684. doi:10.4103/ijdvl.IJDVL_645_17

33. Vineetha M, Sheeja S, Celine MI, et al. Profile of dermatophytosis in a Tertiary Care Center. Indian J Dermatol. 2018;63(6):490-495. doi:10.4103/ijd.IJD_177_18

34. Woldeamanuel Y, Leekassa R, Chryssanthou E, et al. Clinico-mycological profile of dermatophytosis in a reference centre for leprosy and dermatological diseases in Addis Ababa. Mycopathologia. 2006;161(3):167-172. doi:10.1007/s11046-004-3141-5

35. Rezaei-Matehkolaei A, Rafiei A, Makimura K, et al. Epidemiological aspects of dermatophytosis in Khuzestan, southwestern Iran, an Update. Mycopathologia. 2016;181(7-8):547-553. doi:10.1007/ s11046-016-9990-x

36. Kakande T, Batunge Y, Eilu E, et al. Prevalence of dermatophytosis and antifungal activity of ethanolic crude leaf extract of Tetradenia riparia against dermatophytes isolated from patients attending Kampala International University Teaching Hospital, Uganda. Dermatol Res Pract. 2019;2019:9328621. doi:10.1155/2019/9328621
37. Saoji V, Madke B. Efficacy of salicylic acid peel in dermatophytosis. Indian J Dermatol Venereol Leprol. 2019. doi:10.4103/ijdvl. IJDVL $853 \quad 18$

38. Heidrich D, Garcia MR, Stopiglia CDO, et al. Dermatophytosis: a 16year retrospective study in a metropolitan area in southern Brazil. $J$ Infect Dev Ctries. 2015;9(8):865-871. doi:10.3855/jidc.5479

39. Pires CA, Cruz NFSD, Lobato AM, et al. Clinical, epidemiological, and therapeutic profile of dermatophytosis. An Bras Dermatol. 2014;89(2):259-264. doi:10.1590/abd1806-4841.20142569

40. Bhatia VK, Sharma PC. Epidemiological studies on dermatophytosis in human patients in Himachal Pradesh, India. Springerplus. 2014;3:134. doi:10.1186/2193-1801-3-134

41. Vineetha M, Sheeja S, Celine MI, et al. Profile of dermatophytosis in a Tertiary Care Center in Kerala, India. Indian J Dermatol. 2019;64 (4):266-271. doi:10.4103/0019-5154.265814

42. Adefemi SA, Odeigah LO, Alabi KM. Prevalence of dermatophytosis among primary school children in Oke-Oyi community of Kwara state. Niger J Clin Pract. 2011;14(1):23-28. doi:10.4103/11193077.79235

43. Bitew A, Wolde S. Prevalence, risk factors, and spectrum of fungi in patients with onychomycosis in Addis Ababa, Ethiopia: a prospective study. J Trop Med. 2019;2019:3652634. doi:10.1155/2019/3652634

44. Araya S, Tesfaye B, Fente D. Epidemiology of Dermatophyte and Non-Dermatophyte Fungi Infection in Ethiopia. Clin Cosmet Investig Dermatol. 2020;13:291-297. doi:10.2147/CCID.S246183.

\section{Publish your work in this journal}

Clinical, Cosmetic and Investigational Dermatology is an international, peer-reviewed, open access, online journal that focuses on the latest clinical and experimental research in all aspects of skin disease and cosmetic interventions. This journal is indexed on CAS.
The manuscript management system is completely online and includes a very quick and fair peer-review system, which is all easy to use. Visit http://www.dovepress.com/testimonials.php to read real quotes from published authors. 